\title{
PRIVACIDADE E CONFIDENCIALIDADE EM CASOS DE CASAIS DISCORDANTES PARA SÍFILIS*
}

Janina Pontes Pisani ${ }^{1}$, Elma Lourdes Campos Pavone Zoboli², Lislaine Aparecida Fracolli

\begin{abstract}
RESUMO: Estudo exploratório que buscou, junto a usuários da Liga de Combate à Sífilis e outras Doenças Sexualmente Transmissíveis do Hospital das Clínicas da Universidade de São Paulo suas percepções acerca da privacidade e confidencialidade em situação hipotética com casal heterossexual, quando um dos parceiros tem sífilis, e compará-las às percepções encontradas em estudos realizados com profissionais da Estratégia Saúde da Família e "Potenciais usuários do Sistema Único de Saúde". A coleta de dados, realizada através de entrevista semiestruturada, perguntava como o profissional deveria agir numa situação em que um dos parceiros com sífilis não quer revelar o fato à esposa, porém pede que se realize o exame diagnóstico e o tratamento, sem o conhecimento de sua mulher. Foram entrevistados 32 usuários, e a análise dos dados mostrou que existe aceitação da participação do profissional no processo de revelação da verdade, e aproximações entre as percepções dos diversos grupos estudados.
\end{abstract}

PALAVRAS-CHAVE: Bioética; Privacidade; Comunicação sigilosa; Sífilis.

\section{PRIVACY AND CONFIDENTIALITY IN SYPHILIS-DISCORDANT COUPLES}

ABSTRACT: This exploratory study sought the perceptions of users of the Combat on Syphilis And Other SexuallyTransmitted Diseases group of the Clinical Hospital of the University of São Paulo on issues of confidentiality and privacy in a hypothetical situation involving a heterosexual couple one of whom has syphilis, and compared them with the findings of studies carried out by health professionals from the Family Health Strategy and 'potential users of the Public Health System'. The data collection, carried out via semi-structured interview, asked how the health professional should act in a situation where a partner with syphilis does not want to reveal the fact to his wife, and who wishes to be tested and treated without his wife's knowledge. 32 service users were interviewed, and data analysis showed that there is acceptance of the participance of the health professional in the process of revealing the truth and that there are similarities among the different groups studied.

KEYWORDS: Bioethics; Privacy; Secret communication; Syphilis.

\section{PRIVACIDAD Y CONFIDENCIALIDAD EN CASOS DE PAREJAS DISCORDES PARA SÍFILIS}

RESUMEN: Estudio exploratorio que ha buscado, junto a usuarios de la Liga de Combate à Sífilis y otras Enfermedades Sexualmente Transmisibles del Hospital de Clínicas de la Universidad de São Paulo sus percepciones acerca de la privacidad y confidencialidad en situación hipotética con pareja heterosexual, cuando uno de los compañeros tiene sífilis, y compararlas a las percepciones de estudios realizados con profesionales de la Estrategia Salud de la Familia y "Potenciales usuarios del Sistema Único de Salud". Los datos fueron recogidos por medio de entrevista semiestructurada, en la cual se preguntaba cómo el profesional debería actuar en una situación en que uno de los compañeros con sífilis no revela el hecho a la esposa, pero pide que se realize el exámen diagnóstico y el tratamiento, sin el conocimiento de su mujer. Fueron entrevistados 32 usuarios, y el análisis de los datos reveló que existe aceptación de la participación del profesional en proceso de revelación de la verdad, y aproximaciones entre las percepciones de los diversos grupos estudiados.

PALABRAS CLAVE: Bioética; Privacidad; Comunicación sigilosa; Sífilis.

*Pesquisa financiada com bolsa de iniciação científica CNPq.

${ }^{1}$ Enfermeira.

${ }^{2}$ Enfermeira. Pós-Doutora em Bioética. Professora do Departamento de Enfermagem em Saúde Coletiva e do Programa de PósGraduação em Enfermagem da Universidade de São Paulo.

${ }^{3}$ Enfermeira. Pós-Doutora. Professora Departamento de Enfermagem em Saúde Coletiva e do Programa de Pós-Graduação em Enfermagem da Universidade de São Paulo.

Autor correspondente:

Janina Pontes Pisani

Universidade de São Paulo

Av. Engenheiro Antônio Heitor Eiras Garcia, 1621 - 05588-0010 - São Paulo-SP-Brasil

Recebido: 20/02/11

E-mail: janina.pisani@usp.br

Aprovado:16/05/11 


\section{INTRODUÇÃO}

Dentre os problemas éticos que surgem na atenção à saúde é comum a preocupação frente a dois princípios: a privacidade e a confidencialidade. A Carta dos Direitos dos Usuários de Saúde, do Ministério da Saúde, assegura que deve ser exercido o respeito à confidencialidade de toda e qualquer informação pessoal ${ }^{(1)}$.

A privacidade é o direito de decidir quais informações pessoais serão mantidas sob controle e em quais condições estas informações podem ser reveladas ${ }^{(2)}$. No contexto da saúde, a privacidade permite que o paciente revele à equipe de saúde situações potencialmente embaraçosas, num ambiente em que a privacidade estará assegurada pela manutenção do segredo ${ }^{(3)}$. Já a confidencialidade é a garantia da preservação das informações dadas em confiança e a proteção contra a sua revelação não autorizada $^{(4)}$. A confidencialidade é uma responsabilidade dos profissionais para com os pacientes ${ }^{(5)}$.

A privacidade e a confidencialidade relacionam-se com a intimidade das pessoas. Tradicionalmente, a intimidade apresentou-se bastante vinculada às crenças religiosas e às atitudes morais das pessoas. Porém, ao longo do século XX, com o reconhecimento político e cultural do indivíduo, há uma maior sensibilidade para os temas da intimidade e privacidade que resulta em um novo modo de gestão da vida, da morte, do corpo e da sexualidade. Este novo modo de gestão confere maior importância à autonomia dos sujeitos nas decisões morais, com mais respeito aos direitos de intimidade e privacidade ${ }^{(6)}$.

A significativa transformação na compreensão da intimidade resultou na defesa de que esta é inerente a todo ser humano, convertendo-se em um direito humano fundamental; a confidencialidade passa a representar o dever que preserva o caráter privativo de cada ser humano ${ }^{(6)}$.

Embora a confidencialidade constitua um princípio essencial na relação profissional de saúde/paciente, há situações em que o seu não cumprimento é justificável, como é o caso da possibilidade de causar danos à saúde ou à segurança da coletividade, ou de terceiros identificáveis ${ }^{(7)}$.

Levando em consideração o contexto das Doenças Sexualmente Transmissíveis (DST), conflitos dessa ordem podem surgir quanto ao dever do profissional de revelar o diagnóstico do paciente a terceiros diretamente envolvidos e que têm sua saúde potencialmente sob risco ${ }^{(8)}$. Alguns pacientes recusam-se a revelar a seus parceiros sexuais a ocorrência de uma DST, colocando-os em situação de risco. O profissional, nesse caso, deve escolher entre preservar a privacidade do paciente ou proteger a saúde do(a) sua parceiro(a), uma decisão nem sempre fácil de ser tomada.

A sífilis é uma doença infecciosa crônica que acomete praticamente todos os órgãos e sistemas e, apesar de ter tratamento eficaz e de baixo custo, ainda é considerada um problema de saúde pública ${ }^{(9)}$. Por esse motivo, é importante que sejam feitos o rastreamento e a notificação de parceiros das pessoas diagnosticadas com sífilis, para que estes também realizem tratamento e não sofram as possíveis consequências da doença.

Em geral, a notificação de parceiros pode ser feita pelo próprio paciente (o paciente avisa seus parceiros para procurar tratamento médico) ou pelo profissional (o profissional contata os parceiros do paciente e indica $o$ diagnóstico e tratamento) ${ }^{(10)}$. Ainda não é claro qual dessas estratégias se mostra mais eficaz, mas há evidências de que a notificação pelo profissional pode ser mais eficiente na captação de parceiros ${ }^{(11)}$.

Profissionais da saúde, quando confrontados em situações como essa, sentem falta de uma política que defina um modo correto de agir ${ }^{(12)}$. Em estudo realizado com profissionais da Estratégia Saúde da Família (ESF) perguntou-se como agiriam na situação em que um paciente com sífilis não quer revelar este fato a sua esposa, porém pede que se realize o exame diagnóstico e o tratamento, se necessário, sem o conhecimento de sua mulher. A resposta destes profissionais apontou para diferentes cursos de ação, mas preponderou a preservação da privacidade do paciente sobre a proteção da saúde da parceira $^{(2)}$, deixando para aquele a responsabilidade de revelar a verdade.

Tendo como base o estudo mencionado acima, foi elaborado um questionário de múltipla escolha e aplicado junto aos funcionários da Escola de Enfermagem da Universidade de São Paulo (EEUSP) que se enquadravam no perfil de "potenciais usuários do Sistema Único de Saúde - SUS" ${ }^{(13)}$. A pesquisa mostrou que há algumas aproximações entre o curso de ação que o profissional tomaria e o que o "potencial usuário do SUS" elegeria como o mais adequado. Também pudemos notar que os sujeitos preferiram que a revelação da doença fosse feita pelo próprio paciente, não cabendo ao profissional revelar à mulher sobre a doença de seu companheiro. Este ponto de vista também foi encontrado de maneira hegemônica entre os profissionais da $\mathrm{ESF}^{(2)}$.

Dando continuidade aos dois estudos anteriores ${ }^{(2,13)}$, a presente pesquisa teve por objetivo identificar junto a pacientes da Liga de Combate à Sífilis e outras Doenças Sexualmente Transmissíveis do Hospital das Clínicas da Faculdade de Medicina da Universidade de São Pau- 
lo (HCFMUSP) pontos de vista acerca da privacidade e confidencialidade em situação hipotética com casal heterossexual, quando um dos parceiros tem sífilis; e confrontar os pontos de vista encontrados com as alternativas recomendadas pelos profissionais do ESF e pelos "potenciais usuários do SUS" entrevistados em estudos anteriores, que apresentavam cenário hipotético similar.

\section{METODOLOGIA}

Trata-se de um estudo exploratório, quantiqualitativo. Foram sujeitos do estudo pacientes diagnosticados com sífilis e em tratamento na Liga de Combate à Sífilis e outras DST. Por se tratar de um estudo exploratório, utilizou-se o critério de amostra incidental não probabilística.

A Liga de Combate à Sífilis e outras DST é uma entidade de caráter científico e assistencial, com o objetivo de tratar pacientes, promover cursos sobre temas da área e realizar campanhas de prevenção.

A coleta de dados foi feita durante o mês de junho de 2009, por meio de entrevistas norteadas por um questionário elaborado com base nos cursos de ação sugeridos por profissionais da ESF e utilizando o mesmo cenário hipotético ${ }^{(2)}$. O formulário foi o mesmo utilizado no estudo feito com "potenciais usuários do SUS"(13). Dentre as alternativas de como o profissional poderia agir (cursos de ação) o respondente deveria escolher a que mais se aproximasse do seu ponto de vista.

Havia, ainda, espaço para comentários e sugestão de outra forma de como lidar com o problema. O caso e a questão apresentados aos participantes foram:

O senhor M tem sífilis. Ele não quer contar o que tem para sua esposa, mas quer protegê-la da doença. Enquanto está em tratamento, pede à equipe que faça o exame em sua companheira sem que ela saiba. O que você esperaria dos profissionais de Saúde da Família envolvidos neste caso?

a) Que solicitassem o exame somente depois do marido contar a verdade para a mulher;

b) Que solicitassem o exame sem contar a verdade para a mulher, mas estimulando que o marido o fizesse;

c) Que solicitassem o exame sem contar a verdade para a mulher, mas estimulando que o marido o fizesse se o resultado fosse positivo;

d) Que solicitassem o exame sem contar a verdade para a mulher e sem estimular que o marido o fizesse; e) Que solicitassem o exame sem contar a verdade para a mulher, se o relacionamento extraconjugal fosse um episódio fortuito, caso contrário, que estimulassem o marido a contar a verdade para a esposa;

f) Nenhuma das alternativas anteriores. Neste caso, o que esperariam?

O participante foi convidado a contribuir com o estudo durante a espera pela consulta, em um ambiente que garantisse sua privacidade, e o termo de consentimento livre e esclarecido foi devidamente assinado naquele momento. A pesquisa foi aprovada pelo Comitê de Ética em Pesquisa do HCFMUSP através do protocolo n. 0206/09, e está dentro das normas éticas para pesquisa.

Foram coletados dados demográficos como idade, sexo, religião e estado civil, que serviram como variáveis independentes para os testes de associação estatística. Os dados referentes ao estado civil e religião foram coletados por meio de questões abertas. Para facilitar o tratamento estatístico, as respostas foram agrupadas segundo a classificação e critérios utilizados no Censo Demográfico $2000^{(14)}$.

Para a análise quantitativa usou-se o programa Epi-Info v.3.4.1, SPSS v.11.5. Considerou-se um valor de $\mathrm{p}<0,05$ para determinação de significância estatística. Para a análise qualitativa utilizou-se a análise de conteúdo segundo Bardin ${ }^{(15)}$.

\section{RESULTADOS}

\section{Perfil dos respondentes}

Para a coleta de dados, foram realizadas 32 entrevistas. A faixa etária predominante foi a de 51 a 60 anos, correspondendo a $31,25 \%$ dos sujeitos, seguidas pelas faixas de 41 a 50 anos $(28,13 \%)$ e 31 a 40 anos $(15,62 \%)$. A maioria dos respondentes era do sexo masculino $(65,62 \%)$, sendo apenas 11 dos sujeitos mulheres.

Quanto ao estado civil, a maioria dos entrevistados $(56,25 \%)$ referiu ser casada. Os solteiros representaram $28,12 \%$ dos sujeitos, e apenas dois entrevistados referiram ser viúvos. Relativo à religião, a maioria dos entrevistados (53,13\%) referiu ser católica, 9 dos participantes referiram ser batistas, e três usuários referiram não seguir nenhuma religião específica.

\section{Cursos de ação escolhidos}

No quadro 1 temos a distribuição dos sujeitos, segundo a alternativa escolhida, dentre as opções dadas de como o profissional poderia agir (cursos de ação) frente o caso e a questão apresentados aos participantes. 
Quadro 1 - Distribuição dos sujeitos segundo a opção de curso de ação escolhida. São Paulo, 2009

\begin{tabular}{|lcc|}
\hline Alternativa & $\mathbf{N}$ & $\mathbf{\%}$ \\
Alternativa A & 15 & 46,88 \\
Alternativa B & 1 & 3,12 \\
Alternativa C & 5 & 15,63 \\
Alternativa D & 4 & 12,50 \\
Alternativa E & 1 & 3,12 \\
Alternativa F & 6 & 18,75 \\
\hline Total & 32 & 100,00 \\
\hline
\end{tabular}

Para a análise estatística dos dados realizamos o teste do Qui-Quadrado e o teste exato de Fisher, associando as alternativas escolhidas e as variáveis idade, sexo, estado civil e religião, mas nenhum dos testes mostrou associação estatística significativa $(\mathrm{p}>0,05)$. Isto pode ser devido às limitações da amostra, que foi pequena e não aleatória.

Para reconhecer as aproximações e distanciamentos entre as posturas dos profissionais do ESF, as expectativas dos "ponteciais usuários do SUS" e as dos usuários da Liga de Combate à Sífilis e outras DST, comparamos os resultados do presente estudo com os obtidos em cada estudo anterior $^{(2,13)}$, o que está disposto no quadro 2.

Quadro 2 - Distribuição das opções de curso de ação escolhidas segundo "Potenciais Usuários do SUS", Profissionais da ESF e Usuários da Liga de Combate à Sífilis. São Paulo, 2009

\begin{tabular}{|ccccccc|}
\hline Alternativa escolhida & "Potenciais Usuários do SUS" & \multicolumn{2}{c|}{ Profissionais da ESF } & \multicolumn{2}{c|}{ Usuários da Liga } \\
\hline A & $\mathbf{N}$ & $\mathbf{\%}$ & $\mathbf{N}$ & $\mathbf{\%}$ & $\mathbf{N}$ & $\mathbf{\%}$ \\
B & 15 & 34,09 & 9 & 69,24 & 15 & 46,88 \\
C & 14 & 31,82 & 1 & 7,69 & 1 & 3,12 \\
D & 7 & 15,91 & 1 & 7,69 & 5 & 15,63 \\
E & 1 & 2,27 & 1 & 7,69 & 4 & 12,50 \\
F & 1 & 2,27 & 1 & 7,69 & 1 & 3,12 \\
\hline Total & 6 & 13,64 & - & - & 6 & 18,75 \\
\hline
\end{tabular}

Além disso, também totalizamos os resultados obtidos nos três estudos para ter um panorama geral das alternativas mais escolhidas (Quadro 3).

Quadro 3-Distribuição das opções de curso de ação escolhidas nos estudos com Profissionais da ESF, "Potenciais usuários do SUS" e Usuários da Liga de Combate à Sífilis. São Paulo, 2009

\begin{tabular}{|lcc|}
\hline Alternativa & $\mathbf{N}$ & $\mathbf{\%}$ \\
Alternativa A & 39 & 43,82 \\
Alternativa B & 16 & 17,98 \\
Alternativa C & 13 & 14,61 \\
Alternativa D & 6 & 6,74 \\
Alternativa E & 3 & 3,37 \\
Alternativa F & 12 & 13,48 \\
\hline Total & 89 & 100,00 \\
\hline
\end{tabular}

A questão da veracidade é algo valioso para os usuários da Liga de Combate à Sífilis, como exemplifica este discurso:

A verdade é valiosa. A mentira só traz prejuizo, a mulher tem que saber. O marido deve contar. (Usuário 17)
Os sujeitos manifestam sua necessidade de apoio por parte dos profissionais, para contar a verdade a seus companheiros:

Caso a mulher seja muito "esquentada" o profissional deve ajudar a revelar a verdade. (Usuário 14)

Muitos dos entrevistados, ao escolher como a mais adequada a alternativa que mencionava o apoio para contar a verdade, fizeram ressalvas, sugerindo que o profissional de saúde deveria aguardar para que o marido revelasse a doença para sua companheira:

Se o marido não quiser contar, o profissional deve contar e proteger a mulher. (Usuário 20)

O marido não deve esconder, devia contar. Se não, o profissional pode tomar a frente e contar. (Usuário 27)

Este mesmo ponto de vista também foi encontrado em um novo curso de ação sugerido na alternativa $\mathrm{F}$ :

Que estimulasse o marido a contar, caso não queira, o profissional pode contar. A mulher tem que se proteger. (Usuário 18) 
Há quemespere do profissional uma cumplicidadena situação, mesmo que para isso, o profissional tenha que mentir. Os trechos de discurso a seguir exemplificam este ponto de vista:

Caso o marido não queira, o profissional deve preservar o direito dele de não contar, até para que o marido possa confiar mais no profissional. Mas a mulher deve fazer o exame mesmo assim. (Usuário 28)

Que [o profissional] solicitasse o exame e contasse para a mulher o motivo. (Usuário 3)

Acho que o profissional deve contar à mulher o que tá acontecendo, porque o marido jamais vai chegar e contar para a mulher, e ela tem o direito de saber. (Usuário 13)

Caso o marido não queira contar, o profissional deve contar à esposa antes de solicitar o exame. (Usuário 21)

\section{DISCUSSÃO}

A alternativa "esperam que a equipe de saúde solicite o exame somente depois que o marido contar a verdade para sua mulher" foi o curso de ação mais escolhido pelos entrevistados da Liga de Combate à Sífilis, representando 46,88\%. Esta alternativa também foi a mais escolhida entre os profissionais daESF${ }^{(2)}(69,24 \%)$, e"potenciais usuários do SUS"(13) (34,09\%), representando $43,82 \%$ dos sujeitos incluídos nos três estudos.

Esta escolha parece indicar uma valorização do princípio da veracidade, vista tanto pelos usuários como pelos profissionais como um direito que, se negligenciado, pode causar danos.

O usuário, ao se deparar com o diagnóstico de uma DST, vivencia diversos sentimentos e preocupações, e deixar para ele a responsabilidade de revelar sobre a doença aos possíveis parceiros pode representaruma fonte de preocupação. Assim, parece que o respeito à autonomia não pode significar abandono e o profissional deve auxiliar e oferecer apoio ao usuário no momento da revelação da verdade, para que ele se sinta confiante e amparado ao fazê-lo. Ainda assim, os usuários esperam que o profissional lhes dê um tempo para contar e, se não conseguirem, admitem que a equipe intervenha.

A alternativa "esperam que a equipe de saúde solicite o exame sem contar a verdade para a mulher, e estimulem o marido a contá-la" foi escolhida por apenas um dos entrevistados da Liga de Combate à Sífilis (3,12\%). Ao compararmos com as pesquisas anteriores, notamos que entre os profissionais da ESF esta alternativa também foi escolhida por apenas um profissional (7,69\%). Já entre os "potenciais usuários do SUS", esta alternativa foi a segunda mais escolhida, representando

\section{$31,82 \%$ dos sujeitos.}

A diferença na escolha da referida alternativa poderia ser explicada pelo fato do grupo dos profissionais da ESF e dos usuários da Liga de Combate à Sífilis já terem vivenciado a situação apresentada na entrevista. Muitos pacientes que passam pela experiência de ter uma DST vivenciam sentimentos negativos, como tensão e perda de confiança entre o casal, medo, raiva, vergonha e diminuição da autoestima ${ }^{(16)}$.

Poderíamos dizer que, para os entrevistados, no momento em que se descobriram como possíveis agentes de um dano a terceiros, a preservação da saúde prevaleceu como um valor superior a sua própria intimidade.

A alternativa "esperam que a equipe de saúde solicite o exame sem contar a verdade para a mulher, e estimulem o marido a contá-la se o resultado dela for positivo" foi escolhida por $15,62 \%$ dos entrevistados.

Em caso do resultado do exame ser positivo, parece haver mais critérios para a quebra da confidencialidade, pois a probabilidade de outro dano maior ocorrer é bastante provável se a doença não for tratada, e ainda mais sabendo-se que existe a probabilidade concreta de se minimizar os danos iminentes através do diagnóstico precoce e tratamento da sífilis.

A alternativa "esperam que a equipe de saúde solicite o exame sem contar a verdade para a mulher e sem estimular o marido para contá-la" foi escolhida por $12,5 \%$ dos entrevistados. Entre os "potenciais usuários do SUS" e profissionais da ESF esta alternativa foi escolhida por apenas um participante em cada estudo.

A solicitação de um exame sem o conhecimento da própria pessoa é prejudicial, pois somente através do conhecimento de seu estado ela poderá mudar o comportamento e impedir a contaminação de outros ou a sua própria reinfecção. A maior porcentagem de escolha desta alternativa entre os usuários da Liga de Combate à Sífilis pode refletir a vivência negativa da experiência de ser diagnosticado com uma DST, levando ao medo e insegurança em revelar o seu diagnóstico.

A alternativa $\mathrm{F}$, que era aberta para o usuário elaborar sugestões para a resolução do caso, foi escolhida por seis dos entrevistados (18,75\%). Em sua maioria, eles sugerem uma maior participação do profissional na revelação do diagnóstico, além da necessidade de um atendimento individualizado, considerando as particularidades de cada casal e de cada situação.

Parece que a divisão de responsabilidades, ou até mesmo uma corresponsabilização entre o profissional de saúde e o usuário, garantem um ambiente de suporte e apoio para que a experiência de estar com uma DST seja vivida de maneira menos sofrida.

Na prática das profissões da saúde o segredo sempre foi considerado um dever profissional de excepcional importância, fato referendado por uma tradição sem quebra, que 
remonta ao Juramento Hipocrático. Em sua origem, o segredo é um dever do profissional e não um direito do usuário; era o médico quem determinava o que se deveria ou não divulgar e, em consequência, o que deveria se calar, mantendo o segredo. Isto diferencia o antigo dever do sigilo do novo direito do usuário à confidencialidade. Agora, o profissional tem um dever porque o usuário tem um direito. Estes direitos geram um dever correlativo para qualquer um que tenha acesso à intimidade ou à privacidade de outrém, seja ou não profissional de saúde ${ }^{(6)}$.

\section{CONCLUSÃO}

Após a análise dos dados, pudemos perceber que, embora a privacidade e a confidencialidade envolvendo casais soro-discordantes sejam temas que podem causar problemas éticos para os envolvidos, os sujeitos abordados nas pesquisas parecem convergir para um mesmo caminho de conciliação dos valores em conflito.

Tanto os usuários como os profissionais de saúde parecem buscar um caminho intermediário para solucionar a questão, ou seja, tentam conciliar o respeito à privacidade do marido com a revelação da verdade para a mulher, o que permite realizar na prática os dois valores.

Além disso, a maioria dos entrevistados tende a acreditar que a presença de uma DST representa um risco potencial à saúde de terceiros, a ponto de justificar a quebra da confidencialidade. Parece, assim, que consideram a confidencialidade não como um dever absoluto, mas como um dever que admite exceções em casos específicos, quando outros valores mais importantes ficam ameaçados ou são lesados com o cumprimento estrito do respeito à confidencialidade.

Os usuários esperam a participação e apoio do profissional neste momento de seus tratamentos. Assim, o profissional de saúde deve estar atento e analisar cada situação individualmente, identificando em que casos ele deve intervir, e como deve ser esta intervenção, sempre buscando oferecer apoio e favorecer uma melhor vivência da situação.

\section{REFERÊNCIAS}

1. Ministério da Saúde (BR). Secretaria de Atenção à Saúde. Carta dos direitos dos usuários da saúde. Brasília: Ministério da Saúde; 2006. Série legislação de saúde.

2. Zoboli ELCP. Bioética e atenção básica: um estudo de ética descritiva com enfermeiros e médicos do programa saúde da família [tese]. São Paulo(SP): Universidade de São Paulo; 2003.
3. Loch JA Confidencialidade: natureza, características e limitações no contexto da relação clínica. Bioética. 2003;11(1):51-64.

4. Francisconi CF, Goldim JR. Aspectos bioéticos da confidencialidade e privacidade. In: Costa SIB, Oselka G, Garrafa V. Iniciação à bioética. Brasília: Conselho Federal de Medicina; 1988. p. 269-85.

5. Massarollo MCKB, Saccardo DP, Zoboli ELCP. Autonomia, privacidade e confidencialidade. In: Oguisso T, Zoboli E, organizadores. Ética e bioética: desafios para a enfermagem e a saúde. Barueri: Manole; 2006. p. 111-35.

6. Gracia D. Como arqueros al blanco: estudios de bioética. Madrid: Triacastela; 2004.

7. Sacardo DP, Fortes PAC. Desafios para a preservação da privacidade no contexto da saúde. Bioética. 2000;8(2):307-22.

8. Carvalho FT, Rocha KB, D’Elia P, Fontoura VA. Revelação do diagnóstico de doenças sexualmente transmissíveis a parceiros(as) sexuais: um dilema bioético? DST: JBras Doenças Sex Transm. 2005;17(3):219-24.

9. Avelleira JCR, Bottino G. Sífilis: diagnóstico, tratamento e controle. Rev Bras Dermatol. 2006; 81(2):111-26.

10. Fleetwood J. STDs in patients with multiple partners: confidentiality. Am Fam Physician. 2006;74(11):1963-4.

11. Catherine M, Coetzee N, Zwarenstein M, Lombard C, Guttmacher S, Oxman AD, et al. Strategies for partner notification for sexually transmitted diseases. Cochrane Database of Systematyc Reviews. In: The Cochrane Library, Issue 03, Art. No. CD002843. DOI: 10.1002/14651858. CD002843.pub4.

12. Rivas JD, Sulmasy DP. Sexually transmited disease: a private matter? Am Fam Physician. 2002;66(7):1351-5.

13. Pisani JP, Zoboli ELCP. Doenças sexualmente transmissíveis: preservar a confidencialidade do marido ou proteger a saúde da mulher? Cogitare Enferm. 2009;14(3):476-83.

14. Instituto Brasileiro de Geografia e Estatística (IBGE). Metodologia do censo demográfico 2000. Rio de Janeiro: IBGE; 2003.

15. Bardin L. Análise de conteúdo. Lisboa: Edições 70; 2006.

16. Ford CA, Jaccard J, Millstein SG, Viadro CI, Eaton JL, Miller WC. Young adult's attitudes, beliefs, and feelings about testing for curable STDs outside of clinic settings. J Adolesc Health. 2004;34(2):266-9. 